\title{
IMPLEMENTATION AND PERFORMANCE OF A TAU LEPTON SELECTION WITHIN THE ATLAS TRIGGER SYSTEM AT THE LHC
}

A. dos Anjos, S. Armstrong, J.T. Baines, C.P. Bee, M. Biglietti, A. Bogaerts, M. Bosman, B. Caron, P. Casado, G. Cataldi, D. Cavalli, G. Comune, P. Conde, G. Crone, D. Damazio, A. De Santo, M. Diaz Gómez, A. Di Mattia, N. Ellis,

D. Emeliyanov, B. Epp, S. Falciano, H. Garitaonandia, S. George, V. Ghete, R. Goncalo, J. Haller, S. Kabana, A. Khomich, G. Kilvington, J. Kirk,

N. Konstantinidis, A. Kootz, A.J.. Lankford, A. Lowe, L. Luminari, T. Maeno, J. Masik, C. Meessen, A.G. Mello, R. Moore, P. Morettini, A. Negri, N. Nikitin, A. Nisati, C. Osuna, C. Padilla, N. Panikashvili, F. Parodi, E. Pasqualucci, V. Perez-Reale, J.L. Pinfold, P. Pinto, Z. Qian, S. Resconi, S. Rosati, C. Sánchez, C. Santamarina, D.A. Scannicchio, C. Schiavi, E. Segura, J M. de Seixas, S. Sivoklokov, A. Sobreira, R. Soluk, E. Stefanidis, S. Sushkov, M. Sutton, S. Tapprogge, S. Tarem, E. Thomas, F. Touchard, G. Usai, B. Venda Pinto, A. Ventura, V. Vercesi, T. Wengler, P. Werner, S.J. Wheeler, F.J. Wickens, W. Wiedenmann, M. Wielers, H. Zobernig

THE ATLAS HIGH LEVEL TRIGGER GROUP * presented by P. CASADO ${ }^{\dagger}$

IFAE, Edifici Cn, Bellaterra, E-08193, Spain

\begin{abstract}
The ATLAS experiment at the Large Hadron Collider (LHC) has an interaction rate of up to $10^{9} \mathrm{~Hz}$. The trigger must efficiently select interesting events while rejecting the large amount of background. The First Level trigger will reduce this rate to around $O(75 \mathrm{kHz})$. Subsequently, the High Level Trigger (HLT), comprising the Second Level trigger and the Event Filter, will reduce this rate by a factor of $O\left(10^{3}\right)$. Triggering on taus is important for Higgs and SUSY searches at the LHC. In this paper tau trigger selections are presented based on a lepton trigger if the tau decays leptonically or via a dedicated tau hadron trigger if the tau disintegrates semileptonically. We present signal efficiency with the electron trigger using the data sample $A \rightarrow \tau \tau \rightarrow$ ehadron, and rate studies obtained from the dijet sample.
\end{abstract}

*http://atlas.web.cern.ch/atlas/groups/daqtrig/hlt/authorlists/como2005.pdf †Work supported by the Institut de Física d'Altes Energies (IFAE) and Universitat Autònoma de Barcelona 


\section{Introduction}

The Large Hadron Collider(LHC) is expected to start data taking in 2007 at CERN (European Organisation for Nuclear Research). Proton-proton collisions will be produced at a center of mass energy of $14 \mathrm{TeV}$ and design luminosity of $10^{34} \mathrm{~cm}^{-2} \mathrm{~s}^{-1}$.

ATLAS is a multipurpose detector which will have to cover a wide variety of aspects of high energy physics phenomenology : from discovering new physical phenomena to performing precision measurements. In order to achieve this task, the detector is composed of different tracking subdetectors, a solenoid, electromagnetic (e.m.) and hadronic calorimeters, muon subdetectors and a toroid, as well as the Trigger and Data AcQuisition systems $(\mathrm{TDAQ})^{1}$.

\section{The ATLAS Trigger system}

(1) The ATLAS Trigger system is organised in three levels. The first level (LVL1) is hardware-based and has to reduce the input rate from 40 $\mathrm{MHz}$ to $75 \mathrm{kHz}$ in less than $2.5 \mu \mathrm{s}$. The result of the LVL1 selection contains information about the type of trigger and position of the possible particle candidates that cause the event to be accepted. After a positive LVL1 decision the data are transferred from electronic pipeline memories to distributed buffers in so called Read-Out Systems (ROSs). The second (LVL2) and third (Event Filter -EF-) levels are software based systems running on linux PC farms. They are referred collectively as High Level Trigger (HLT). They use full granularity data of all detectors and can also combine information from different detectors. The LVL2 accesses a few percent of the total detector data in a Region of Interest (RoI) provided by the LVL1 result by direct network request to the ROSs. This reduces the event rate to about $2 \mathrm{kHz}$. After a positive LVL2 decision the complete event is assembled and made available to the EF, which has access to the full detector data and the latest calibration and alignment information. Events accepted by the HLT are stored on tape for further analysis. The goal is to achieve an average decision time of $10 \mathrm{~ms}$ and $1 \mathrm{~s}$ for LVL2 and $\mathrm{EF}$, respectively, although the system could easily scale to accommodate larger execution times if needed.

(2) The HLT selection software ${ }^{2}$ has been designed with Object Oriented methodology and is implemented in $\mathrm{C}++$. It can run in the LVL2, EF and offline environments. This is possible because the differences between the environments are hidden by well defined interfaces and the same framework 
$\left(\right.$ Athena $\left.^{3}\right)$ is used.

The HLT selection software is subdivided into four main sub-packages: (a) The Steering organizes the processing of the HLT algorithms. (b) The Event Data Model (EDM) covers all data entities in the event. (c) The Data Manager provides the means for accessing the event data during the trigger processing. (d) The HLT Algorithms can either reconstruct the event or check hypothesis about previously calculated quantities.

(3) The data access of the HLT algorithms is a fundamental part in the design of the HLT architecture. The HLT algorithm requests data in a RoI using the offline transient data store (Athena Storegate ${ }^{4}$ ) as interface and the Region of Interest package, a software component which returns Storegate pointers to the data inside the specified detector geometrical region. These data are in form of $\mathrm{C}++$ objects, convenient for the algorithm (e.g. calorimeter cells which hold energy and position), contrary to the data coming from the detector electronics which is in raw format (i.e. a binary file with the read-out channel information).

\section{Tau selection}

The selection of taus is important at the LHC for the discovery of new particles. Taus appear in decay modes with significant branching fractions, for example in charged Higgs, in $A \rightarrow \tau \tau$ ( $A$ being the pseudo-scalar Higgs from the Minimal Supersymmetric Standard Model). In this paper we focus on $A \rightarrow \tau \tau \rightarrow$ ehadron which represents $25 \%$ of the $A \rightarrow \tau \tau$ production.

The tau can decay via two modes: leptonically, with one electron or muon in the final state (we only consider here the electron decay); or semileptonically, with hadrons in the final state.

In both cases the HLT selection procedure follows the Region of Interest mechanism. The data request is restricted to a region with different size for the various trigger.

In the case of the tau decaying into an electron, one can use the single isolated electron trigger of ATLAS. At LVL1 calorimeter information is used with a segmentation in trigger towers of $0.1 \times 0.1$ in $\Delta \eta \times \Delta \phi$. A cluster is defined with the two most energetic pairs of e.m. towers (adjacent in eta or phi) from the central $2 \times 2$ core of the $4 \times 4$ window. An isolation cut is applied in the outer region of the $4 \times 4$ region and in the hadronic calorimeter. At LVL2, the energy and position measurements obtained at LVL1 are refined; the leakage into the hadronic calorimeter is evaluated and variables related to the shower shape in the e.m. calorimeter are used to perform preliminary particle identification. If a candidate is found to be 

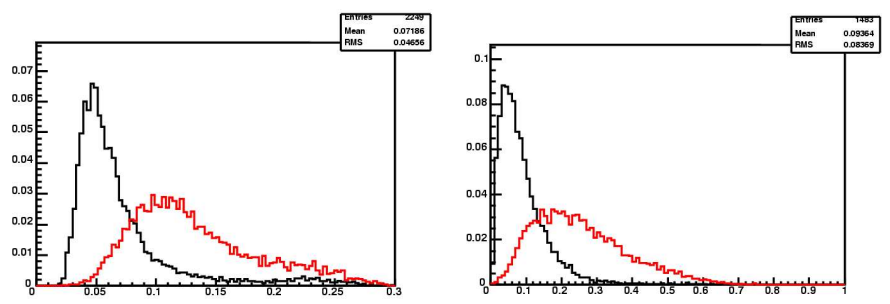

Figure 1. E.m. radius (left) and isolation fraction (right) for taus decaying hadronically (black) and jets (red).

consistent with an electron, track reconstruction is performed in the Inner Detector (ID); cluster to track association is done using $(\eta, \phi)$ matching criteria, achieving further rejection against fake candidates; in case the matching was successful, the $E_{T} / p_{T}$ ratio between the transverse energy measured in the e.m. calorimeter and the transverse momentum of the corresponding ID track is evaluated for particle identification. If the objects under analysis fulfill the required signatures, the event and its LVL2 result are passed to the EF, where more precise clustering algorithms can be performed, and where information on the complete event is available, along with more precise calibrations and alignment constants.

In the case of tau decaying into hadrons one has to use the genuine tau trigger, used in conjunction with missing $E_{T}$ at $p_{T} \approx 35 \mathrm{GeV}$, and standalone for $p_{T} \approx 60 \mathrm{GeV}$. At LVL1 the cluster is defined from the e.m. towers as in the electron case and in addition, the inner core of the hadronic calorimeter is used. The isolation region is defined from the outer part of the $4 \times 4$ window of the e.m. and the hadronic calorimeters. $E_{T}$ of the cluster can be higher than for the electron trigger while the applied isolation cut is looser. The selection of LVL2 and EF has as a guidance the offline. In this case two of the variables with highest rejection power are depicted in Fig. 1. The rejection factor obtained using the whole selection is 200 for an efficiency of $50 \%$. At LVL2 we are using previously considered variables and new quantities imported from the offline. The list is the following: (1) Total energy $\left(E_{T}\right)$ calculated in an inner and outer region for the e.m. and the hadronic calorimeters. (2) The e.m. radius: energy weighted radius of the cluster calculated in the e.m. calorimeter. The distribution of this variable for the offline is shown in Fig. 1 (left). (3) The width in the energy deposition: energy weighted standard deviation in $\eta$. (4) The isolation fraction: fraction of energy deposited in a region of 


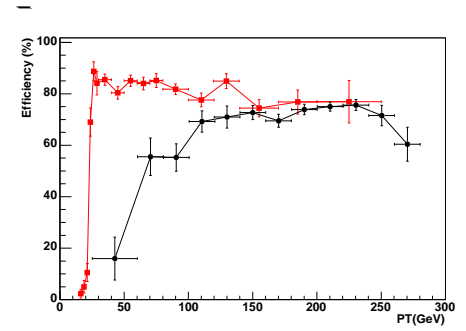

Figure 2. Efficiency of the electron trigger as a function of the $P_{T}$ of the electron (red) and as a function of the $P_{T}$ of the tau (black).

$0.1<R<0.2$. The distribution of this variable for signal and background is also shown in Fig. 1 (right).

\section{Results}

The sample $A \rightarrow \tau \tau \rightarrow$ e hadron at low luminosity with electronic noise and pile up has been used to calculate efficiencies of the isolated electron trigger as a function of $p_{T}$ of the electron and of $p_{T}$ of the $\tau$, normalizing with respect to events having an offline reconstructed electron. This is shown in Fig 2. For the electron case, there is the expected sharp rise between 20 and $30 \mathrm{GeV}$ and the maximum value is $\approx 85 \%$. These results confirm those obtained in the collaboration for electrons at $p_{T}=25 \mathrm{GeV}$ and extend them to a broader region in $p_{T}$. For the taus, the slope is much less sharp, the trigger reaches an efficiency plateau for $p_{T}>70 \mathrm{GeV}$. In the lower $p_{T}$ region the electron isolated trigger on taus complements with the hadron tau plus missing $E_{T}$ trigger.

Finally, the HLT rates of two relevant triggers for these studies are estimated using the dijet sample with electronic noise and pile up at low luminosity: for the electron isolated trigger the value is $\approx 32 \mathrm{~Hz}$ and for the tau and missing $E_{T}$ trigger the result is $\approx 5 \mathrm{~Hz}$.

\section{References}

1. ATLAS High-Level Trigger, Data Acquisition and Controls, Technical Design Report, ATLAS TDR-016. ATLAS HLT/DAQ/ DCS Group.

2. "Architecture of the ATLAS online physics-selection software at LHC", Proceedings of the 8th ICATPP Conference on Astroparticle, Particle, Space Physics, Detectors and Medical Applications, 255-259 (2003).

3. http://atlas. web.cern.ch/Atlas/GROUPS/SOFTWARE/OO/architecture

4. The StoreGate: a Data Model for the Atlas Software Architecture, Proceedings CHEP03, La Jolla, California, March 24-28, 2003. 